Article

\title{
Mechanical Properties and Adhesion of a Micro Structured Polymer Blend
}

\section{Brunero Cappella}

Federal Institute for Material Research and Testing (BAM), VI.4, Unter den Eichen 87, Berlin 12205, Germany; E-Mail: brunero.cappella@bam.de

Received: 31 May 2011; in revised form: 5 July 2011 / Accepted: 14 July 2011 /

Published: 15 July 2011

\begin{abstract}
A 50:50 blend of polystyrene (PS) and poly(n-butyl methacrylate) (PnBMA) has been characterized with an Atomic Force Microscope (AFM) in Tapping Mode and with force-distance curves. The polymer solution has been spin-coated on a glass slide. PnBMA builds a uniform film on the glass substrate with a thickness of $\cong 200 \mathrm{~nm}$. On top of it, the PS builds an approximately $100 \mathrm{~nm}$ thick film. The PS-film undergoes dewetting, leading to the formation of holes surrounded by about $2 \mu \mathrm{m}$ large rims. In those regions of the sample, where the distance between the holes is larger than about $4 \mu \mathrm{m}$, light depressions in the PS film can be observed. Topography, dissipated energy, adhesion, stiffness and elastic modulus have been measured on these three regions (PnBMA, PS in the rims and PS in the depressions). The two polymers can be distinguished in all images, since PnBMA has a higher adhesion and a smaller stiffness than PS, and hence a higher dissipated energy. Moreover, the polystyrene in the depressions shows a very high adhesion (approximately as high as PnBMA) and its stiffness is intermediate between that of PnBMA and that of PS in the rims. This is attributed to higher mobility of the PS chains in the depressions, which are precursors of new holes.
\end{abstract}

Keywords: polymer blends; dewetting; mechanical properties; adhesion; AFM 


\section{Introduction}

The technological and industrial relevance of polymer blends has been at the origin of a large interest in the mechanisms of phase separation in bulk polymer blends, which are nowadays quite well known and understood.

If the polymers are confined to thin films, i.e., if the surface area to volume ratio is large, interface effects may govern the phase separation, and the behavior of thin polymer films may be substantially different from that of bulk samples [1]. Furthermore, in case of thin films, the phenomenon of dewetting plays a major role in determining the morphology of the film. Dewetting [2,3] occurs depending on the balance between the thickness of the melt polymer or of the solution used for the film preparation, the liquid/vapor surface tension and the density of the liquid. Dewetting is much more probable for thin films than for bulk samples.

The dewetting processes in thin films are primarily of two kinds, the heterogeneous nucleation and the spinodal dewetting [2,3]. Both mechanisms lead to the creation of holes. In the first case, the formation of the holes is initiated by the presence of impurities and by residual stresses in the film, and the holes are uncorrelated; in the second case, the holes are the result of surface undulations originated by thermal fluctuations and have a correlation length depending on the film-substrate interactions.

Consequences of the hole-formation process are the creation of uncovered surfaces on the substrate and the accumulation of the removed material in rims or shoulders at the borders of the holes.

The holes grow with time until they merge and disrupt the continuous film [4], resulting in ribbons of polymer that become unstable via Rayleigh instability and build droplets.

If the film is prepared by spin-coating, the film morphology is usually "frozen" in one of the described stages, far from thermodynamic equilibrium. This can be understood as follows. A polymer blend solution with low polymer concentration is deposited onto a substrate. At the beginning of the spin coating process most of the solution is cast off leaving a thin layer on the substrate. As the solvent evaporates, the polymer concentration increases significantly. This leads to a considerable increase of the viscosity of the solution, resulting in a reduced mobility of the polymer chains, till the films become glassy. The poor mobility of the chains hinders the slippage necessary for the formation of holes and ribbons [3]. In other words, the rapid increase of the polymer concentration in solution is analogous to a temperature quench [5].

Due to the rapid quench of the polymer blend, the sample is frozen in non-equilibrium morphology. The size of the lateral domains is determined by the complex interaction between the rapid quench and the continuously decreasing mobility. Hence, the final morphology of the blend depends on various factors, such as temperature [6,7], the blend composition [8-10] and the molecular weight of polymers [11,12], the kind of solvent [7,8] and of substrate[11], and the film thickness [9,11,13,14].

The mechanical properties [15] and the adhesion [16] of such micro- and nanostructured samples have been very seldom investigated. Yet, this is the field where AFM finds its most useful application, since it is the only instrument able to measure local properties with high force and lateral resolution. 


\section{Materials and Methods}

The granulate of poly(n-butyl methacrylate) (PnBMA) was purchased from Scientific Polymer Products (Ontario, NY) and was used without further purification. The molecular weight, the polydispersity index, and the glass transition temperature given by the supplier are $M_{\mathrm{W}}=320 \mathrm{kDa}$, $M_{\mathrm{W}} / M_{\mathrm{N}} \leq 2.58$, and $T_{\mathrm{g}}=22^{\circ} \mathrm{C}$.

Polystyrene (PS) was purchased from BDH Chemicals (Poole, England); the molecular weight, the polydispersity index, and the glass transition temperature are $M_{\mathrm{W}}=100 \mathrm{kDa}, M_{\mathrm{W}} / M_{\mathrm{N}} \leq 3.05$ and $T_{\mathrm{g}}=98^{\circ} \mathrm{C}$.

The mechanical behavior of PnBMA, both in bulk [17,18] and as thin or ultrathin films [19], and of PS $[18,20]$ is well known and was examined in detail by means of force-displacement curves in our previous experiments.

The glass slides were cleaned and rinsed with toluene. The same amount of both polymers $(32.3 \mathrm{mg})$ was dissolved in $2 \mathrm{~mL}$ toluene. A polymer film was spin coated from $500 \mu \mathrm{L}$ of the solution with a velocity of $\omega=2,000 \mathrm{rpm}$ for $1 \mathrm{~min}$. Care was taken to ensure that the film covered the whole glass slide evenly. To ensure that equilibrated and solvent-free films were obtained, the samples were dried for 2 weeks under ambient conditions.

Atomic force microscopy (AFM) measurements were performed with a MFP-3D microscope (Asylum Research, Santa Barbara, CA). For all measurements the AFM was equipped with a Pointprobe NCL cantilever (Nanosensor, Wetzlar-Blankenfeld, Germany) with elastic constant $k_{\mathrm{c}}=54 \pm 1 \mathrm{~N} / \mathrm{m}$ and a silicon tip with an estimated radius of $R=35 \pm 5 \mathrm{~nm}$. The elastic constant has been determined from the noise spectrum [21].

\section{Results and Discussion}

Figure 1 (A) shows a $40 \times 40 \mu \mathrm{m}^{2}$ topography picture of the blend, acquired in Tapping Mode. We can observe a non-uniform PS film, presenting several holes, on top of the PnBMA film. The holes are due to phase separation and dewetting: the more hydrophilic PnBMA wets the glass substrate, while PS stays on the surface, but, due to its immiscibility with PnBMA, minimizes the contact surface with PnBMA by tearing apart and forming holes [5,7]. PS would form small droplets on top of the PnBMA film, if the phase separation was not "frozen" by the solvent quench, as discussed in the introduction. The bottom of the holes is not an even PnBMA surface, since small droplet-like structures of PS stay inside the holes. Also, these small domains are to be ascribed to the rapid solvent evaporation during spin-coating. Initially, the polymers in both phases still have sufficient mobility to diffuse over the several micron large distances separating the phases. As the solvent evaporates, the amount of the minority component in both phases decreases and mass transport over the larger and larger distances can no longer take place due to the reduction of the polymer diffusion. As a result, the small droplets inside the holes are formed [7]. 
Figure 1. Topography (A) and phase-shift (B) image of a $40 \times 40 \mu \mathrm{m}^{2}$ area of the poly(n-butyl methacrylate) (PnBMA)/polystyrene (PS) blend, acquired in Tapping Mode. The white and black line in both pictures indicates the position of the line profile shown in panel (C). PnBMA (darker in panel A and brighter in panel B) forms a film in contact with the glass substrate; PS (brighter in panel A and darker in panel B) stays on top of PnBMA and, due to the immiscibility of the two polymers, forms holes to minimize the contact surface. The phase shift is higher on PnBMA than on PS, due to larger adhesion and compliance. The bottom of the holes present several droplet-like PS particles, two of which are indicated by the second and third arrow on the right in panel C. The phase shift on such particles is very large, due to topography artifacts. The PS film presents several depressions (one is indicated by the first arrow on the left in panel $\mathrm{C}$ ), on which the phase shift is similar to that on PnBMA.
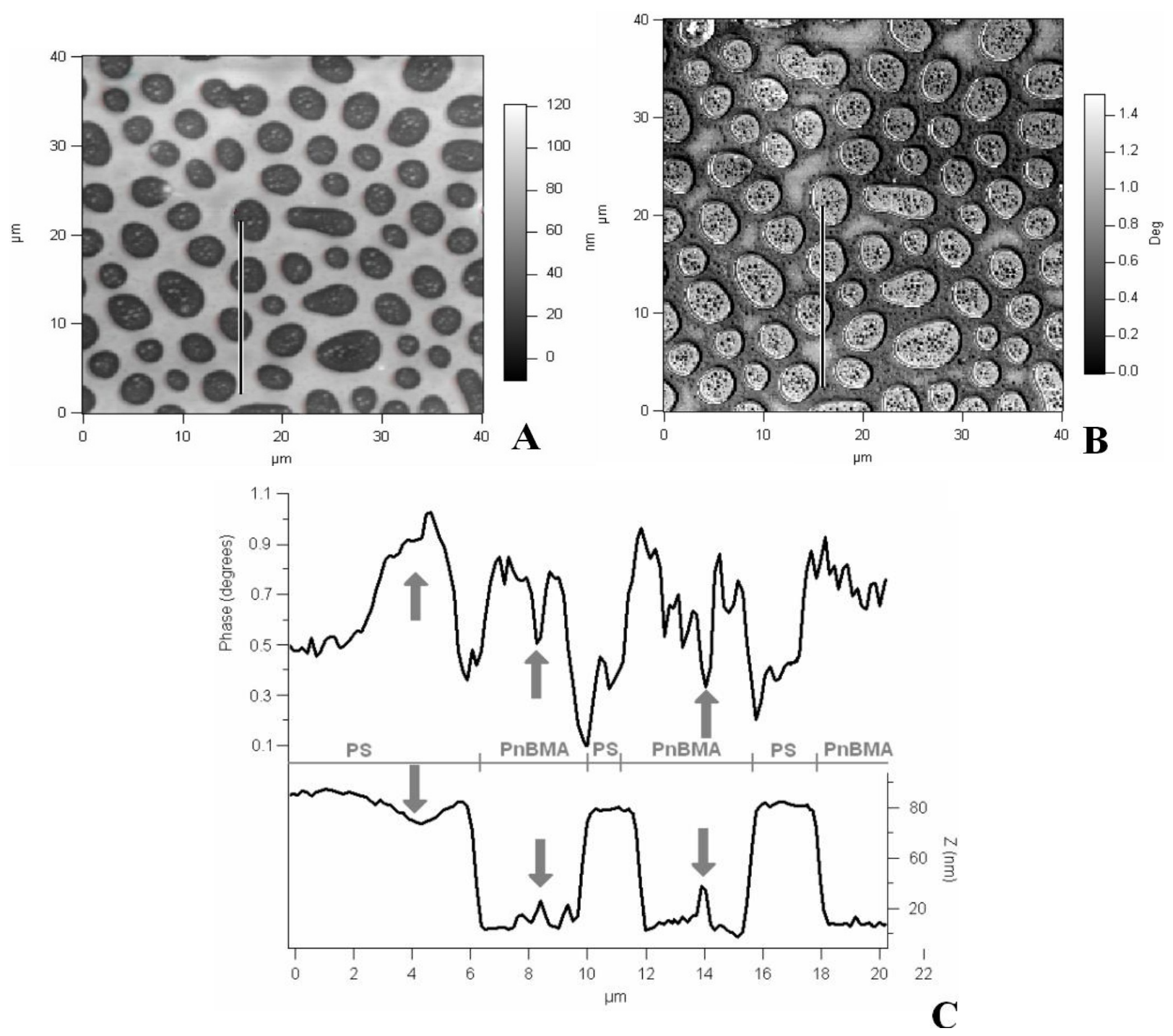

The thickness of the whole film (PS + PnBMA) has been measured by scratching the sample and acquiring a topography image in Tapping Mode over the scratch. It resulted to be $300 \pm 10 \mathrm{~nm}$. The thickness of the PS film is $95 \pm 5 \mathrm{~nm}$.

The holes in the PS film have variable dimensions and forms. An analysis of several images, with a total number of 600 holes, has shown that the mean area of the holes is $10 \pm 5 \mu \mathrm{m}^{2}$. The circularity of 
the holes, defined as $p^{2} /(4 \pi A)$, where $p$ is the perimeter and $A$ is the area, is 1.065 . Some holes result from the mergence of two holes; in some cases the outcome of the coalescence is an elongated ellipse (see a hole in the middle of Figure 1), but in other cases the two original holes can still be distinguished (see a hole on the top of Figure 1). In these two cases the circularity is 1.36 and 1.52, respectively. Since the circularity would be 1 for a circle, the form of not merged holes can be assumed to be circular with a mean radius of $1.7 \pm 0.4 \mu \mathrm{m}$.

The corresponding phase shift image is shown in Figure 1(B). The phase shift $\Delta \varphi$ has an offset, so that its minimum value is 0 . The phase shift is due to energy dissipation [22,23]. Hence, the contrast in the corresponding image is due to compliance and adhesion differences between the materials present on the sample surface. Figure 1(B) clearly shows that the phase shift is smaller on the PS film (darker) than on the PnBMA film (brighter). The PS droplets inside the holes can be distinguished, too. The image also shows small regions inside the PS film, most of all in the upper part of the image, where $\Delta \varphi$ is considerably higher than on the rest of the PS surface.

Figure 1(C) shows a line profile of both images (the position is indicated in Figure $1(\mathrm{~A}, \mathrm{~B})$ by a white and black line). From left to right (i.e., from bottom to top in Figure 1(A,B)), we see three PS regions $(80 \mathrm{~nm}<Z<95 \mathrm{~nm}$ and $0.35<\Delta \varphi<0.5)$ and three PnBMA regions $(0 \mathrm{~nm}<Z<15 \mathrm{~nm}$ and $\Delta \varphi \cong 0.8$ ). Also, a typical artifact in phase shift measurements can be observed, namely the fictitious decrease of the phase shift at a very steep edge, like the boundary of the holes. This artifact is due to changes in the tip-sample contact area, affecting both the measured compliance and the tip-sample adhesion. Inside the first and the second hole there are several droplets of PS (two of which are indicated by the last two arrows on the right), causing a sudden decrease of the phase shift. This decrease is due both to material properties and to the steep edges of such particles.

The first arrow on the left indicates a region of the PS film, where $\Delta \varphi$ is larger than on the rest, even than on PnBMA. The $Z$ profile shows that this region is a light depression, 10-20 nm deeper than the residual PS film. Several such depressions can be seen in the phase shift image. Further analysis of the topology of the PS film is necessary to understand the nature of such depressions.

The mean width of the PS film, i.e., the mean distance of neighbor holes, is $1.6 \pm 0.4 \mu \mathrm{m}$. Yet, in some regions the distance of the holes is larger and the PS film is wider. In other words, in some parts of the sample it is possible to inscribe a circle inside the PS film with a radius larger than $2 \mu \mathrm{m}$. In such regions there is always a depression, i.e., a portion of the PS film that is thinner and yields a larger phase shift than the PS film in the $\cong 2 \mu \mathrm{m}$ large rims around the holes. Such depressions have been observed in several works, and their appearance has also been accounted for in simulations [24,25]. Their presence has been explained as a consequence of material flow and thickness fluctuations [26,27]. Also, it has been shown that, when a hole in a dewetting film has reached a certain size, secondary or "satellite" holes appear in the depression [28,29].

The phase signal in such depressions is not affected by the topography, since there are no steep edges and $Z$ changes are very gradual. Hence, the higher phase shift in such depressions must be due to different material properties, i.e., compliance and adhesion. Yet, the contributions of stiffness and adhesion cannot be separated with a measurement of the phase signal. Therefore, force distance curves have been acquired. A force-distance curves measurement is necessary not only for a further understanding of the nature of the depressions, but also for the quantitative characterization of the 
mechanical properties of the PS and PnBMA films and for the comparison between bulk films, homogeneous thin films and the films in the blend.

Figure 2 shows another $60 \times 60 \mu \mathrm{m}^{2}$ region of the sample, both in the topography and in the phase shift image; the insets show the $14 \times 14 \mu^{2}$ area where the force volume measurement has been performed. As in Figure 1, we can see the PS film on top, yielding a smaller phase shift, and the PnBMA surface on the bottom of the holes, yielding a larger phase shift. In the larger sections of the PS film, where the distance between the holes is larger than $\cong 4 \mu \mathrm{m}$, depressions are present. On such depressions, the phase shift signal is comparable with that on the PnBMA film. The force volume measurement has been performed on a very large depression.

Figure 2. Topography (a) and phase-shift (b) image of a $60 \times 60 \mu \mathrm{m}^{2}$ area of the PnBMA/PS blend, acquired in Tapping Mode. The white square indicates the position of the $14 \times 14 \mu \mathrm{m}^{2}$ section, magnified in the insets, where the Force Volume measurement has been performed. This section contains a large depression.

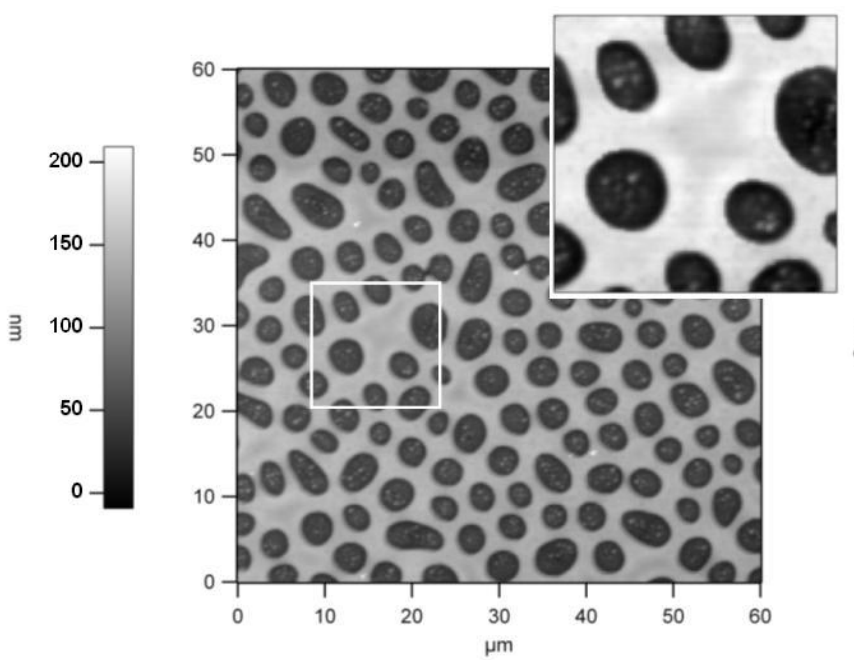

(a)

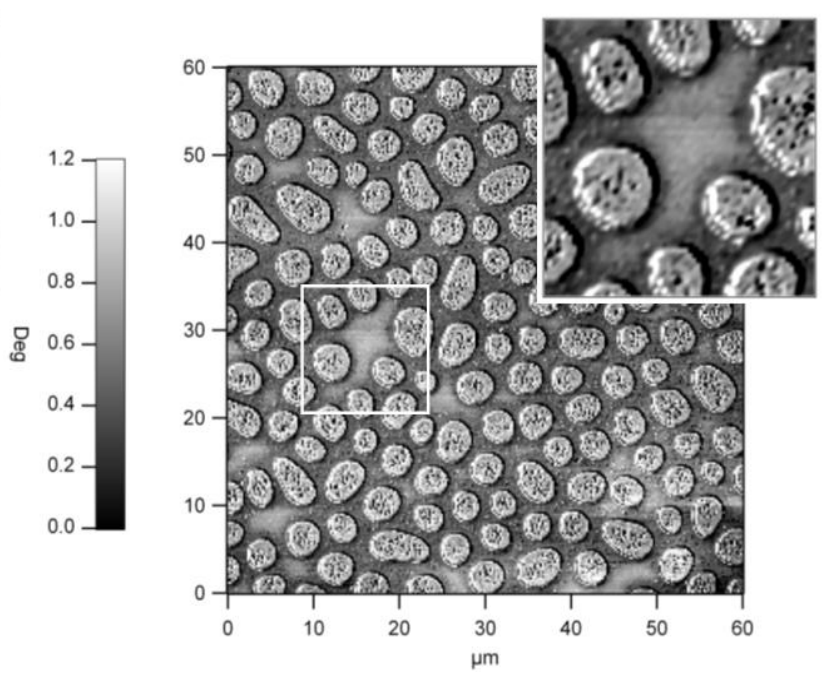

(b)

A force volume measurement is the acquisition of force distance curves on each point of the raster. A force-distance curve [30,31] is a plot of the cantilever deflection $\delta$ versus the piezo displacement $Z$, which defines the sample position relative to the rest position of the cantilever. The applied force $F$ is given by Hooke's law:

$$
F=-k_{c} \delta
$$

where $k_{c}$ is the spring constant of the cantilever.

To acquire a force-displacement curve the sample is approached to the AFM tip up to a maximum force $F_{\max }$ and then withdrawn by means of a piezotransducer, resulting in an approach and a withdrawal curve.

Figure 3 shows two typical force-distance curves of this force-volume measurement acquired on PS (black) and on PnBMA (grey). 
Figure 3. Force-distance curves on PnBMA (grey) and PS (black). The maximum force is $3 \mu \mathrm{N}$. Arrows indicate the direction of motion. PnBMA has a larger adhesion and a lower stiffness than PS.

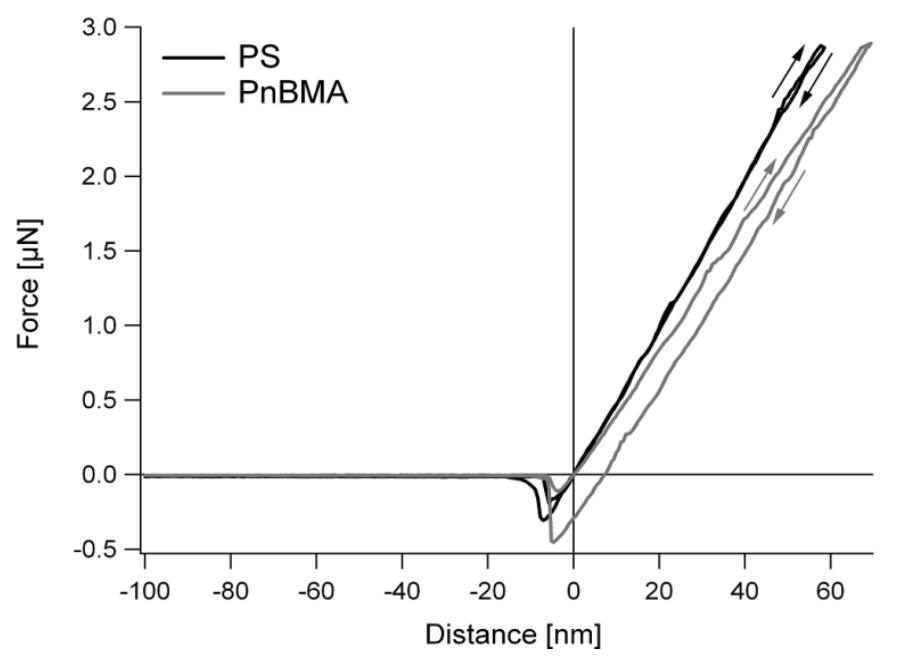

Both the approach and the withdrawal curve are roughly divided in two parts: the zero line (tip and sample are at large distances and do not interact) and the contact line (tip and sample are in contact). In each moiety the two parts are separated by a discontinuity, the jump-to-contact in the approach curve and the jump-off-contact in the withdrawal curve, occurring at the distance where the gradient of the tip-sample attractive (adhesive) forces becomes larger (smaller) than the elastic constant of the cantilever, so that the tip snaps onto the sample or out of contact. The jump-off-contact is hence a measure of the adhesion force between tip and sample.

Looking at Figure 3, it can be seen that the jump-off-contact of the curve on PnBMA is larger than that of the PS curve. Taking advantage of the force volume measurement we can measure the jump-off-contact $F_{\text {adh }}$ on every point of the scanned area. Such a map of the adhesion force is shown in Figure 4(a). It resembles the phase shift image in the bottom inset of Figure 2. The PnBMA regions are brighter than the PS film, i.e., they have a larger adhesion. Also, in force volume measurements, very steep edges engender artifacts due to changes in the contact area [32] or to abrupt movements of the tip, as can be observed at the border of the holes or at the droplet-like PS structures inside the holes.

Moreover, we can see that the depression in the center of the image has a larger adhesion than the rims, almost as large as on PnBMA. This is also shown in the histograms in Figure 4(b). The first histogram (black line) collects all the points in the scanned area. In the other three histograms (bars), the points with artifacts, i.e., the points at the border of the holes and those corresponding to the PS-droplets inside the holes, have been eliminated, and the three regions of the sample have been separated: the PS film is represented by the dark grey bars, the depression and the PnBMA film by the bright grey and white bars. The histograms can be fitted with Gaussian functions. The mean adhesion and the half width at half height are $0.42 \pm 0.08 \mu \mathrm{N}$ for PS in the rims, $0.71 \pm 0.04 \mu \mathrm{N}$ for PnBMA and $0.68 \pm 0.05 \mu \mathrm{N}$ for PS in the depression. 
Figure 4. (a) Adhesion force map of the sample. PnBMA (brighter) has a larger adhesion than PS, but the PS in the depression has an adhesion comparable with that of PnBMA. The adhesion force map resembles the phase shift image (Figure 2(b)). (b) Histograms of the adhesion on the whole area (black line), on the PS film (dark grey bars), on the depression (light grey bars), and on the PnBMA film in the holes (white bars). The histograms of PnBMA, $\left(F_{\text {adh }}=0.71 \pm 0.04 \mu \mathrm{N}\right)$ is separated from that of PS $\left(F_{\mathrm{adh}}=0.42 \pm 0.08 \mu \mathrm{N}\right)$, but not from that of the depression $\left(F_{\mathrm{adh}}=0.68 \pm 0.5 \mu \mathrm{N}\right)$.

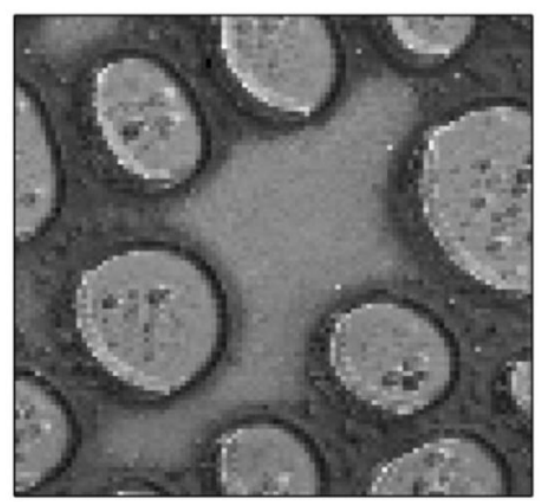

(a)

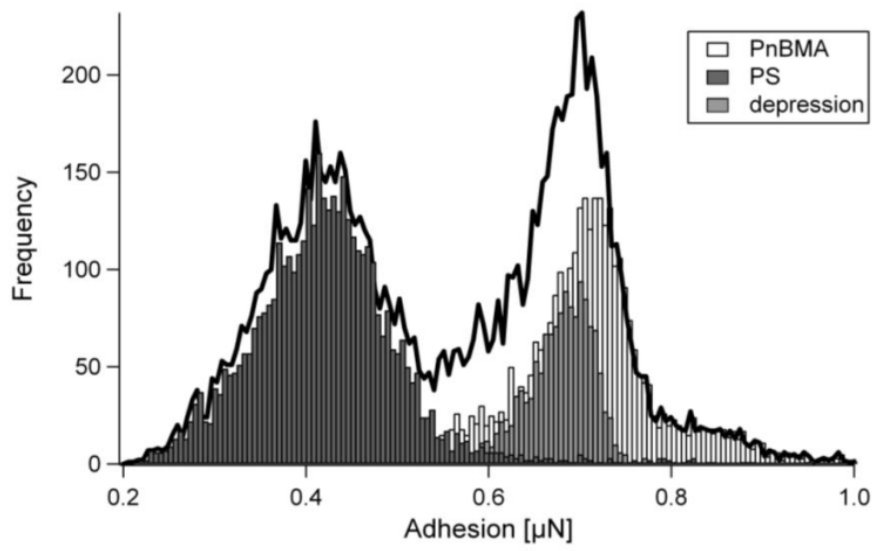

(b)

Along the contact line, the tip eventually indents the sample. The deformation $D$ is given by the difference of the piezo displacement $Z$ and the cantilever deflection $\delta$ :

$$
D=Z-\delta
$$

An important value that can be directly obtained from the force-displacement curves is the spring constant $k_{s}$ of the sample. If the mechanical behavior of both tip and sample is assumed as elastic and can be described by Hooke's law, the balance of forces between sample and probe can be written as:

$$
|\delta| k_{c}=|D| k_{s}
$$

Equations (2) and (3) yield:

$$
k_{c} \delta=\left(\frac{k_{c} k_{s}}{k_{c}+k_{s}}\right) Z
$$

For $k_{\mathrm{s}}>>k_{\mathrm{c}}$, Equation (4) can be approximated with $\delta \cong Z$, showing that, when the cantilever is much more compliant than the sample, the force-displacement curve measures actually the mechanical properties of the cantilever. For $k_{\mathrm{s}}<<k_{\mathrm{c}}$, Equation (4) can be written as:

$$
\delta \cong \frac{k_{s}}{k_{c}} Z
$$

Observing the curves in Figure 3, we can see that the slope of the approach contact line of the PnBMA curve is smaller than that of the PS curve. 
As is the case for adhesion, we can get a stiffness map of the scanned area from the force-volume measurement, shown in Figure 5(a). This image contains the values of $\frac{k_{s}}{k_{c}+k_{s}}$ for each point and, as explained later, is only a rough approximation of the stiffness.

Figure 5. (a) Stiffness map of the sample. PnBMA (darker) is less stiff than PS. The PS in the depression has an intermediate stiffness between that of PnBMA and of PS. (b) Histograms of the stiffness on the whole area (black line), on the PS film (dark grey bars), on the depression (light grey bars), and on the PnBMA film in the holes (white bars). The three histograms are separated $(S=0.89 \pm 0.03$ for PS, $0.85 \pm 0.02$ for the depression and $0.81 \pm 0.04$ for PnBMA).

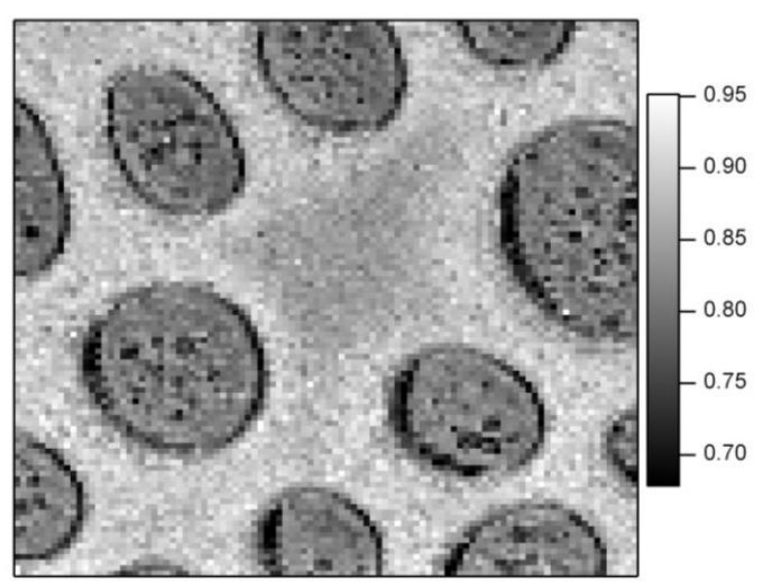

(a)

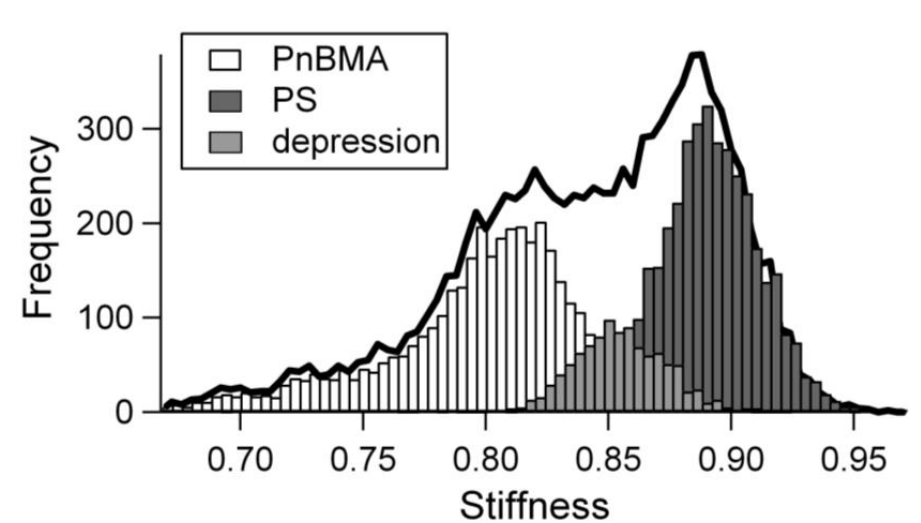

(b)

In this image, the PnBMA regions are darker than the PS film, since the stiffness of PnBMA is considerably smaller than that of PS. The depression in the center can also be clearly seen in the stiffness map, and the stiffness is intermediate between that of PnBMA and the rest of the PS film. The borders of the depression are not as definite as in the adhesion map, and this image does not resemble the phase shift image, since some parts of the depression, which are evident in the bottom inset of Figure 2(b) and in Figure 4, have nearly the same stiffness as the rest of the PS film and cannot be recognized as parts of the depression. The histograms of the stiffness are shown in Figure 5(b). As in Figure 4(b), the black line is the histogram of all points, whereas the dark grey, the bright grey and the white bars are the histograms of the points on the PS film, on the PnBMA film and on the depression, respectively. The fit with Gaussian functions yields a mean stiffness of $0.89 \pm 0.03$ for PS in the rims, $0.81 \pm 0.04$ for PnBMA and $0.85 \pm 0.02$ for PS in the depression. These values can be used to delimit the three domains of the sample, namely the PnBMA film, the PS rims and the depression.

The sample spring constant is obtained by rather crude assumptions and can only be used as an indicator for the sample stiffness. Actually, the mechanical behavior of the sample cannot be described by Hooke's law, since the stiffness $S$ is not a constant, rather a function of the load, given by:

$$
S=\frac{\partial F}{\partial D}=2 a(F) \frac{E}{1-v^{2}}
$$

where $a(F)$ is the radius of the tip-sample contact area, $E$ is the Young's modulus of the sample and $v$ its Poisson's ratio. 
In order to calculate the stiffness $S$ from the measured data it is necessary to express the deformation $D$ or the contact radius $a$ as a function of the applied force $F$. This is provided by the elastic continuum theories, namely Hertz [33], Johnson-Kendall-Roberts (JKR) [34], and Derjaguin-Müller-Toporov (DMT) [35] theories. The Hertz theory neglects the adhesion between tip and sample, which is accounted for in the JKR and DMT theories. The adhesion can be neglected if the applied load is much larger than the adhesion force, which is the case for this measurement.

According to Hertzian theory, if the tip has the form of a half sphere, the deformation and the contact radius are given by:

$$
D=\frac{a^{2}}{R}=\left(\frac{F}{\sqrt{R} K}\right)^{2 / 3}
$$

where $R$ is the tip radius and $K$ the reduced elastic modulus defined as:

$$
\frac{1}{K}=\frac{3}{4}\left(\frac{1-v^{2}}{E}+\frac{1-v_{t}^{2}}{E_{t}}\right)
$$

In Equation (8) $E, E_{\mathrm{t}}, v$ and $v_{\mathrm{t}}$ are the elastic modulus and the Poisson's ratio of tip and sample. The value of the reduced modulus of glass [36] is $80 \mathrm{GPa}$, corresponding to $E=70 \mathrm{GPa}$. The deformation curve on glass, acquired with the same cantilever and fitted with Equation (7), has been used to calibrate the sensitivity $\Omega$ of the AFM [37].

Figure 6 shows the deformation-load curves obtained by averaging the curves in the three domains of the sample, delimited by the values of the stiffness. The three curves can be fitted very well with the Hertz formula for a semispherical tip (Equation (7)). This shows that the assumptions on the tip shape and on the tip-sample adhesion are reasonable. Moreover, it is evident that glass does not influence the mechanical properties of the PnBMA film. This is not surprising considering that the PnBMA film is about $200 \mathrm{~nm}$ thick and the maximum deformation of PnBMA is less than $20 \mathrm{~nm}$. In past measurements of this group [17,38] the mechanical properties of a $190 \mathrm{~nm}$ thick PnBMA films are slightly influenced by the glass substrate, but only for deformations larger than $20 \mathrm{~nm}$. Also the thickness of the PS film is considerably larger than its maximum deformation (95 $\mathrm{nm}$ compared to $9 \mathrm{~nm}$ ) and this explains why its mechanical properties are not influenced by the PnBMA substrate. Such a double-layer effect can be excluded also for the PS in the depression, which is only $10 \mathrm{~nm}$ thinner than the PS in the rims. Moreover, if the PS in the depression was not thick enough to shield the softening effect of the PnBMA substrate, the curve would not have the form given by the Hertz formula (Equation (7)). Rather, the Young's modulus of the PS in the depression would depend on load and deformation, since the effect of the substrate becomes larger and larger with increasing deformation, engendering an apparent decrease of the measured elastic modulus. 
Figure 6. Mean deformation-force curves (grey circles) of PnBMA (top), the depression (middle) and PS (bottom). The curves are fitted with the Hertz formula for a semispherical tip (Equation (7)).

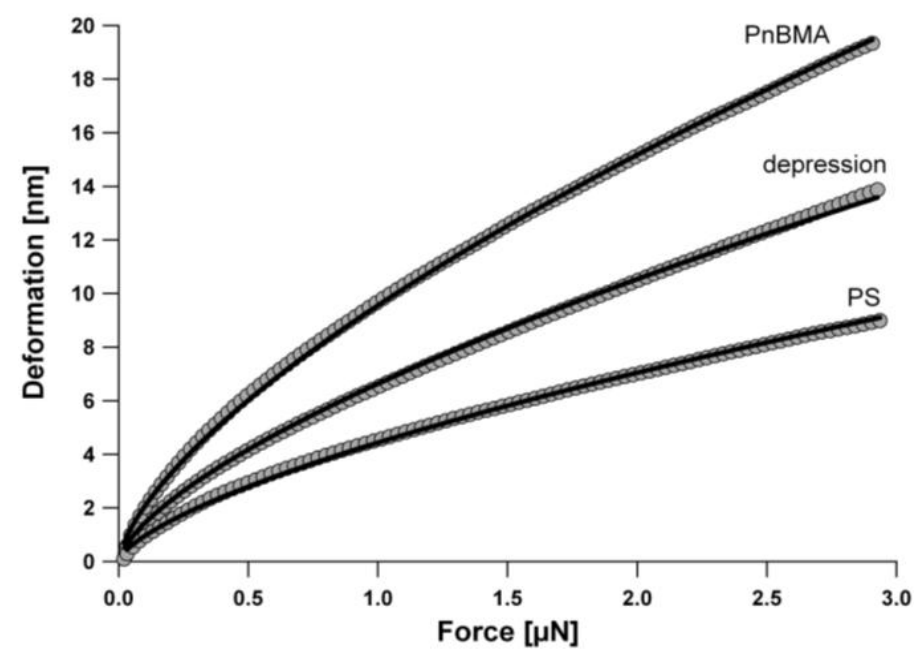

The fit yields a Young's modulus of 3.2 GPa for PnBMA, 10.7 GPa for PS and 5.7 GPa for the depression. In order to ensure the consistency of the data, force-volume measurements have been repeated with the same cantilever on two other samples (prepared in the same way) and on the same sample with three other cantilevers. Only the values of the Young's moduli can be compared, since those of the stiffness and of the adhesion depend on the contact area and on the tip radii, which are different for each cantilever. The mean values of the elastic moduli out of 9 measurements are $3.1 \pm 0.3 \mathrm{GPa}$ for PnBMA, 10.6 $\pm 0.1 \mathrm{GPa}$ for PS and 5.6 $\pm 0.4 \mathrm{GPa}$ for the depression

The elastic moduli of PnBMA and PS measured in this experiment are larger than $1 \mathrm{GPa}$ and $3 \mathrm{GPa}$, i.e., the values previously found by Cappella et al. for bulk polymers [17,18,20], but are in agreement with the measured values of thin films of PnBMA [19,37,38]. Such larger Young's moduli are comprehensible, when the sample preparation and the constraints of the polymers are accounted for. The sample has been prepared through spin-coating, so that the polymer segments have been stretched, causing an increase of the moduli [39]. The fact that PnBMA has the same modulus as spin-coated thin films shows that there are no additional stresses due to the vicinity of PS. For PS, other factors making it stiffer cannot be excluded. The same mechanisms bringing to the formation of large holes are likely to be responsible for inner stresses leading to an increase of the elastic modulus. Moreover, PS is well under its glass transition temperature and the chains cannot relax the inner stresses caused by the sample preparation and the vicinity of PnBMA chains.

The Young's modulus of the sample can also be calculated for each force-distance curve. The resulting map together with the histograms is shown in Figure 7.

PnBMA (dark) has a mean Young's modulus of $3.2 \mathrm{GPa}$. The steep borders of the holes and the droplet-like PS particles inside the holes induce a significant spreading of the relative histogram. Nonetheless, also in this case the histogram of the modulus of PnBMA can be separated from that of PS. Compared to the rest of the PS film, the depression has a considerably smaller value of the Young's modulus. 
Figure 7. (a) Map of the elastic modulus of the sample. PnBMA (darker) has a lower modulus than PS. The PS in the depression has an intermediate modulus between that of PnBMA and of PS. (b) Histograms of the logarithm of the Young's modulus on the whole area (black line), on the PS film (dark grey bars), on the depression (light grey bars), and on the PnBMA film in the holes (white bars). The three histograms are separated $(\log (E)=9.6 \pm 0.15$ for PnBMA, $9.8 \pm 0.13$ for the depression and $10 \pm 0.2$ for PS).

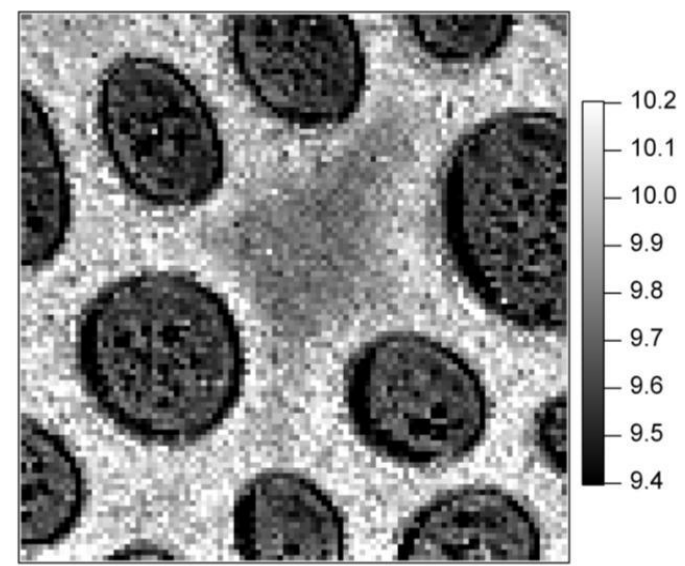

(a)

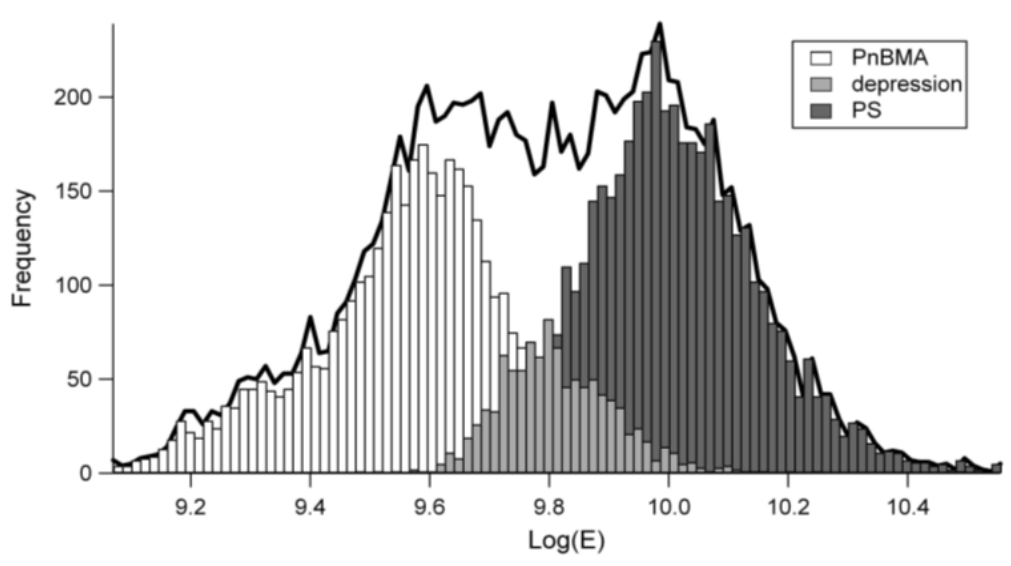

(b)

Observing the maps and the histograms, and in particular the depression, it is clear that the adhesion correlates with the phase shift, but not with the stiffness and the elastic modulus. This is due to the fact that the adhesion and the phase shift of the polymer in the depression are indistinguishable from those of PnBMA, whereas the elastic modulus is intermediate between those of PnBMA and PS. Furthermore, the depression is much smaller in the map of the elastic modulus than in those of the adhesion and of the phase shift, since a large stripe at the border has the same modulus as the PS in the rims, but not the same adhesion and phase shift.

A first important deduction of this observation is that, at least with this system and at this maximum deformation, the dissipated energy and the phase shift are dominated by the adhesion and not by the load-unload hysteresis.

The tip-sample adhesion depends primarily on the interactions between the two materials, but also on the contact area and on the mobility of the sample chains. The interactions between the tip and PS are obviously the same in the rims and in the depression. Hence, they cannot be responsible for differences in the adhesion. The contact area, which is proportional to the square root of the deformation and hence depends on the elastic modulus, is different for the three regions of the sample and influences both the stiffness and the adhesion. Hence, it cannot be responsible for the different dimensions of the depression in the stiffness and adhesion maps.

Yet, it is known that depressions are precursors of new, satellite holes, which would have been formed, if the condition of the film had not been frozen by the rapid solvent evaporation. It can also be assumed that the mobility of the polymer chains in the depressions is higher than that of the chains in the rims, since their position, in contact or near the PnBMA film, is energetically unfavorable.

The stiffness and the elastic modulus are measured along the approach contact line, and in this case the PS chains are pushed to contact with the substrate, i.e., the PnBMA film, whereas the adhesion is 
measured in the withdrawal curve, when the tip is retracted from the sample and the PS chains are pulled away from the PnBMA substrate. Hence, the following can be assumed:

1. The lower stiffness and the higher adhesion of PS in the depression compared to PS in the rims is due to the higher mobility of the chains in the depression;

2. The effect of the mobility on the adhesion is higher than on the stiffness because, when measuring the stiffness, the PS chains are compelled to contact the PnBMA substrate, whereas, when measuring the adhesion, they can follow the tip and detach from the PnBMA substrate.

\section{Conclusions}

The analysis of the 50:50 blend of PnBMA and PS trough AFM in Tapping Mode and in Force Volume mode allows important properties of microstructured polymer films to be ascertained and basic mechanisms of phase separation and dewetting to be elucidated.

When spin-coated, the two polymers undergo phase separation because of their immiscibility. The sample morphology is "frozen" because of rapid solvent evaporation during the spin-coating and presents a PnBMA film on the glass surface and a non-uniform PS film on the top of it, with several holes surrounded by rims.

Measuring the mean deformation as a function of the load and fitting it with the Hertz equation, the Young's modulus of the two polymers could be calculated. The moduli of both polymers are larger than those of molten samples, previously measured in this group. PnBMA has the same modulus as thin films prepared via spin-coating. This indicates that there are no additional stresses due to the vicinity of an immiscible polymer. As for PS, additional stresses due to the glassy state and the vicinity of PnBMA cannot be excluded.

The two polymers can be distinguished in phase signal maps (the dissipated energy on PnBMA is much higher), in adhesion maps (the adhesion with PnBMA is much higher) and in stiffness and/or elastic modulus maps (the stiffness and the Young's modulus of PnBMA are smaller).

Inside the PS film, in regions where the distance between the holes is higher than $\cong 4 \mu \mathrm{m}$, slight depressions can be observed. On these depressions, the phase and the adhesion are as on PnBMA, and the stiffness is intermediate between that of PS in the rims and that of PnBMA. Moreover, the lateral dimensions of the depressions in the phase signal images and in the adhesion images are very similar. This leads to the inference that, at least for this system and at these maximum forces, the dissipated energy and the phase shift are dominated by the adhesion and not by the hysteresis in contact due to plastic deformations.

The larger adhesion and the smaller stiffness are attributed to the higher mobility of the PS chains in the depressions, which in turn is a consequence of the energetic advantage of being not in contact with PnBMA. As a matter of fact, such depressions are precursors of new, satellite holes, which would have formed if the sample morphology were not "frozen" by the solvent evaporation. The chains in the depressions are likely to move away from the PnBMA substrate, and this explains most notably the larger adhesion, since, during a retraction force-distance curve, PS chains have the tendency to follow the tip and to detach from the PnBMA film. 


\section{References}

1. Chung, H.; Wang, H.; Composto, R.J. A morphology map based on phase evolution in polymer blend films. Macromolecules 2006, 39, 153-161.

2. Seemann, R.; Herminghaus, S.; Neto, C.; Schlagowski, S.; Podzimek, D.; Konrad, R.; Mantz, H.; Jacobs, K. Dynamics and structure formation in thin polymer melt films. J. Phys. Condens. Matter 2005, 17, S267-S290.

3. Bäumchen, O.; Jacobs, K. Slip effects in polymer thin films. J. Phys. Condens. Matter 2010, 22, 1-21.

4. Volegova, I.A.; Buzin, A.I. Morphology of ultrathin polymer films based on poly(ethylene oxide) blends. Polym. Sci. A 2007, 49, 1014-1019.

5. Dalnoki-Veress, K.; Forrest, J.A.; Stevens, J.R.; Dutcher, J.R. Phase separation morphology of spin-coated polymer blend thin films. Phys. A 1997, 239, 87-94.

6. Ton-That, C.; Shard, A.G.; Daley, R.; Bradley, R.H. Effects of annealing on the surface composition and morphology of PS/PMMA blend. Macromolecules 2000, 33, 8453-8459.

7. Walheim, S.; Böltau, M.; Mlynek, J.; Krausch, G.; Steiner, U. Structure formation via polymer demixing in spin-cast films. Macromolecules 1997, 30, 4995-5003.

8. Cui, L.; Ding, Y.; Li, X.; Wang, Z.; Han, Y. Solvent and polymer concentration effects on the surface morphology evolution of immiscible polystyrene/poly(methyl methacrylate) blends. Thin Solid Films 2006, 515, 2038-2048.

9. Ton-That, C.; Shard, A.G.; Bradley, R.H. Surface feature size of spin cast PS/PMMA blends. Polymer 2002, 43, 4973-4977.

10. Müller-Buschbaum, P.; Gutmann, J.S.; Stamm, M. Influence of blend composition on phase separation and dewetting of thin polymer blend films. Macromolecules 2000, 33, 4886-4895.

11. Tanaka, K.; Takahara, A.; Kajiyama, T. Surface molecular aggregation structure and surface molecular motions of high-molecular-weight polystyrene low-molecular-weight poly(methyl methacrylate) blend films. Macromolecules 1998, 31, 863-869.

12. Geoghegan, M.; Jones, R.A.L.; Clough, A.S.; Penfold, J. The morphology of as-cast films of a polymer blend-Dependence on polymer molecular-weight. J. Polym. Sci. B 1995, 33, 1307-1311.

13. Tanaka, K.; Takahara, A.; Kajiyama, T. Film thickness dependence of the surface structure of immiscible polystyrene/poly(methyl methacrylate) blends. Macromolecules 1996, 29, 3232-3239.

14. Wang, H.; Composto, R.J. Wetting and phase separation in polymer blend films: Identification of four thickness regimes with distinct morphological pathways. Interface Sci. 2003, 11, 237-248.

15. Reynaud, C.; Sommer, F.; Quet, C.; El Bounia, N.; Duc, T.M. Quantitative determination of Young's modulus on a biphase polymer system using atomic force microscopy. Surf. Interface Anal. 2000, 30, 185-189.

16. Eaton, P.J.; Graham, P.; Smith, J.R.; Smart, J.D.; Nevell, T.G.; Tsibouklis, J. Mapping the surface heterogeneity of a polymer blend: An adhesion-force-distribution study using the atomic force microscope. Langmuir 2000, 16, 7887-7890.

17. Cappella, B.; Kaliappan, S.K.; Sturm, H. Using AFM force-distance curves to study the glass-to-rubber transition of amorphous polymers and their elastic-plastic properties as a function of temperature. Macromolecules 2005, 38, 1874-1881. 
18. Cappella, B.; Kaliappan, S.K. Determination of thermomechanical properties of a model polymer blend. Macromolecules 2006, 39, 9243-9252.

19. Cappella, B.; Silbernagl, D. Nanomechanical properties of mechanical double-layers: A novel semiempirical analysis. Langmuir 2007, 23, 10779-10787.

20. Kaliappan, S.K.; Cappella, B. Temperature dependent elastic-plastic behaviour of polystyrene studied using AFM force-distance curves. Polymer 2005, 46, 11416-11423.

21. Hutter, J.L.; Bechhoefer, L. Calibration of Atomic-Force Microscope tips. Rev. Sci. Instrum. 1993, 64, 1868-1873.

22. Tamayo, J.; Garcia, R. Relationship between phase shift and energy dissipation in tapping-mode scanning force microscopy. Appl. Phys. Lett. 1998, 73, 2926-2928.

23. Garcia, R.; Tamayo, J. Phase contrast and surface energy hysteresis in tapping mode scanning force microsopy. Surf. Interface Anal. 1999, 27, 312-316.

24. Sharma, A. Many paths to dewetting of thin films: Anatomy and physiology of surface instability. Eur. Phys. J. E 2003, 12, 397-407.

25. Neto, C.; Jacobs, K.; Seemann, R.; Blossey, R.; Becker, J. Grün, G. Satellite hole formation during dewetting: Experiment and simulation. J. Phys. Condens. Matter 2003, 15, 3355-3366.

26. Seemann, R.; Herminghaus, S.; Jacobs, K. Shape of a liquid front upon dewetting. Phys. Rev. Lett. 2001, 87, 196101.

27. Herminghaus, S.; Seemann, R.; Jacobs, K. Generic morphologies of viscoelastic dewetting fronts. Phys. Rev. Lett. 2002, 89, 56101.

28. Becker, J.; Grün, G.; Seemann, R.; Mantz, H.; Jacobs, K.; Mecke, K.R.; Blossey, R. Complex dewetting scenarios captured by thin-film models. Nat. Mater. 2003, 2, 59-63.

29. Chen, X.Y.; Lin, J.; Liu, J.M.; Liu, Z.G. Formation and evolution of self-organized hexagonal patterns on silicon surface by laser irradiation in water. Appl. Phys. A 2009, 94, 649-656.

30. Cappella, B.; Dietler, G. Force-distance curves by atomic force microscopy. Surf. Sci. Rep. 1999, 34, 1-104.

31. Butt, H.J.; Cappella, B.; Kappl, M. Force measurements with the atomic force microscope: Technique, interpretation and applications. Surf. Sci. Rep. 2005, 59, 1-152.

32. Mizes, H.A.; Loh, K.G.; Miller, R.J.D.; Ahuja, S.K.; Grabowski, E.F. Submicron probe of polymer adhesion with Atomic Force Microscopy-Dependence on topography and material inhomogeneities. Appl. Phys. Lett. 1991, 59, 2901-2903.

33. Hertz, H.J. Über die Berührung fester elastischer Körper (in German). Reine Angew. Math. 1881, 92, 156-171.

34. Johnson, K.L.; Kendall, K.; Roberts, A.D. Surface energy and contact of elastic solids. Proc. $R$. Soc. London A 1971, 324, 301-313.

35. Derjaguin, B.V.; Müller, V.M.; Toporov, Y.P. Effect of contact deformations on adhesion of particles. J. Colloid Interface Sci. 1975, 53, 314-326.

36. Ullmann's Encyclopedia of Industrial Chemistry; Elvers, B., Hawkins, S., Ravenscroft, M., Rounsaville, J.F., Schulz, G., Eds.; Wiley-VCH: Weinheim, Germany, 1989; p. 386.

37. Cappella, B.; Silbernagl, D. Nanomechanical properties of polymer thin films measured by force-distance curves. Thin Solid Films 2008, 516, 1952-1960. 
38. Silbernagl, D.; Cappella, B. Mechanical properties of thin polymer films on stiff substrates. Scanning 2010, 32, 282-293.

39. Opdahl, A.; Somorjai, G.A. Stretched polymer surfaces: Atomic force microscopy measurement of the surface deformation and surface elastic properties of stretched polyethylene. J. Polym. Sci. B 2001, 39, 2263-2274.

(C) 2011 by the authors; licensee MDPI, Basel, Switzerland. This article is an open access article distributed under the terms and conditions of the Creative Commons Attribution license (http://creativecommons.org/licenses/by/3.0/). 